\title{
Mechanical Properties of 2-D Silica-Silica Continuous Fiber-reinforced Ceramic-matrix Composite Fabricated by Sol-Gel Infiltration
}

\author{
Ha Neul Kim, Dong Jun Kim*, Eul Son Kang** and Do Kyung Kim ${ }^{\dagger}$ \\ Department of Materials Science and Engineering, Korea Advanced Institute of Science and Technology, \\ 373-1 Kusong-dong, Yusong-gu, Daejeon 305-701, Korea \\ *Department of Ceramic Engineering, Yonsei University, 262 Seongsanno, Seodaemun-gu, Seoul 120-749, Korea \\ **Agency for Defense Development, Yusong P.O. Box 35-1, Daejeon, 305-600, Korea
}

(Received June 15, 2009 : Received in revised form July 6, 2009 : Accepted July 13, 2009)

\begin{abstract}
Continuous Fiber-reinforced Ceramic-matrix Composites (CFCCs) were fabricated by a sol-gel infilitration method that has a changing processing condition, such as the repetitions of infilitration. In order to investigate the relationship between the processing condition and the mechanical properties of composites, the mechanical properties of specimens were measured by means of a 4-point flexural strength test while the evidence of strength degradation were microstructurally characterized. There seemed to be a minimum density value that existed at which the delamination between the fabrics would not occur. In the case that the density of silica CFCCs exceeded $1.55 \mathrm{~g} / \mathrm{cm}^{3}$, the flexural strength also exceeded approximately $18 \mathrm{MPa}$ at least. By applying the Minimum Solid Area (MSA) analysis of the porous structure, the correlation between the relative density and the mechanical properties of composites will be discussed.
\end{abstract}

Key words silica, continuous fiber-reinforced ceramic-matrix composites (CFCCs), mechanical properties, Minimum solid area analysis.

\section{Introduction}

Amorphous silica has been considered as the appropriate ceramic which can be applied on the radome and antenna window materials which need the transparency of microwave, the excellent thermal conductivity and the mechanical properties such as fracture toughness and erosion resistance. ${ }^{1-3)}$ Amorphous silica has high melting temperature and superior thermal shock resistance to other structural ceramics such as nitrides, borides and carbides, and also has low dielectric constant and loss tangent in the range of microwave frequency. However, monolithic silica bulk is not appropriate to be used on the structural application directly because it has inferior strength and fracture toughness to several structural ceramic materials. Thus, it is necessary to improve mechanical properties of monolithic silica so that it can be accepted for the structural applications. One of the means of achieving improved mechanical properties is by using either two- or three-dimensional networks of continuous fibers as reinforcements to the ceramic-matrix material,

Corresponding author

E-Mail : dkkim@kaist.ac.kr (D. K. Kim) that leads to newer structural materials, known as continuous fiber-reinforced, ceramic-matrix composites (CFCCs). CFCCs have a potential to overcome the drawback of brittleness through effective matrix microcracking, significant extent of fiber/matrix debonding and finally, fiber pull-out and fiber breakage. ${ }^{4-6)}$

It is difficult to achieve fully dense CFCCs with sol-gel method, so that the porosities are inevitable in the fabricated CFCCs. Thus, it is important to investigate the dependence of the mechanical properties on the porosities in the CFCCs. The minimum solid area (MSA) analysis developed by $\mathrm{Rice}^{7)}$, is the analytical prediction of mechanical properties for porous materials, which have various porous structures such as stacked solid particles and stacked pores and so on. The MSA analysis on the porous CFCCs has been studied by a few of researchers. Fisher et al. $^{8)}$, and Jamet et al. ${ }^{9)}$ reported that the relationship between the porosity and the mechanical properties of SiC-SiC fibrous composites was consistent with MSA model.

In the present paper, we report the mechanical properties of 2-D silica-silica CFCCs fabricated by sol-gel infiltration method. The relationship between the relative density and the mechanical properties is discussed via MSA analysis 
and we support the description on the relationship through microstructural analysis with scanning electron microscope (SEM).

\section{Experimental procedure}

High purity silica fabric is woven 2-dimensionally in $5 \mathrm{H}$ satin from the yarns which consist of 1000 2000 silica filaments with $8 \sim 10 \mathrm{~mm}$ thickness and the purity of fabric is over $99.9 \%$. The thickness of the fabric is approximately $1.2 \mathrm{~mm}$. The matrix material is chosen as Ludox ${ }^{\circledR}$ LS (silica sol, DuPont, USA). The silica sol is the colloidal suspension in which $30 \mathrm{wt} \%$ amorphous silica particles $(\sim 12 \mathrm{~nm})$ is distributed. The unit surface area is $215 \mathrm{~m}^{2} / \mathrm{g}$, the $\mathrm{pH}$ is 8.2 and the viscosity is $8 \mathrm{cP}$ at $25^{\circ} \mathrm{C}$, and the weight ratio of $\mathrm{SiO}_{2} / \mathrm{Na}_{2} \mathrm{O}$ is approximately 270 which is the highest $\mathrm{SiO}_{2}$ content among the DuPont products. To determine the temperature of heat-treatment, thermal gravitational analysis (TGA) was conducted on both the silica fabric and the silica sol.

The silica fabric was cut as the size of $80 \mathrm{~mm} \times$ $80 \mathrm{~mm}$, stacked 7 times, and infiltrated with silica sol by means of vacuum-infiltration device which can reduce pores in the matrix. After being dried at $60{ }^{\circ} \mathrm{C}$, The heattreatment was conducted in the box furnace under air atmosphere. The mechanical properties of silica composites, such as the flexural strength, strain, and elastic modulus, were characterized by means of 4-point bending test which was referred from flexural properties of continuous fiber-reinforced advanced ceramic composites in ASTM C1341-00. The samples used for flexural test were rectangular bars of $10 \mathrm{~mm}$ width, $7 \mathrm{~mm}$ thickness and $45 \mathrm{~mm}$ length. The span length was fixed at $26 \mathrm{~mm}$ outer span and $13 \mathrm{~mm}$ inner span and loading speed was $0.5 \mathrm{~mm} / \mathrm{min}$. In order to characterize the bonding state between the fabric and the matrix, the fracture surfaces of the specimen resulted from flexural test were observed by SEM. Because the strength of silica CFCCs depends on the content and the structure of porosity strongly, the minimum solid area (MSA) analysis was performed to describe the relationship between the relative density and the mechanical properties of silica CFCCs.

\section{Results and discussions}

\subsection{Thermal gravitational analysis (TGA)}

It is important to analyze the material state varying

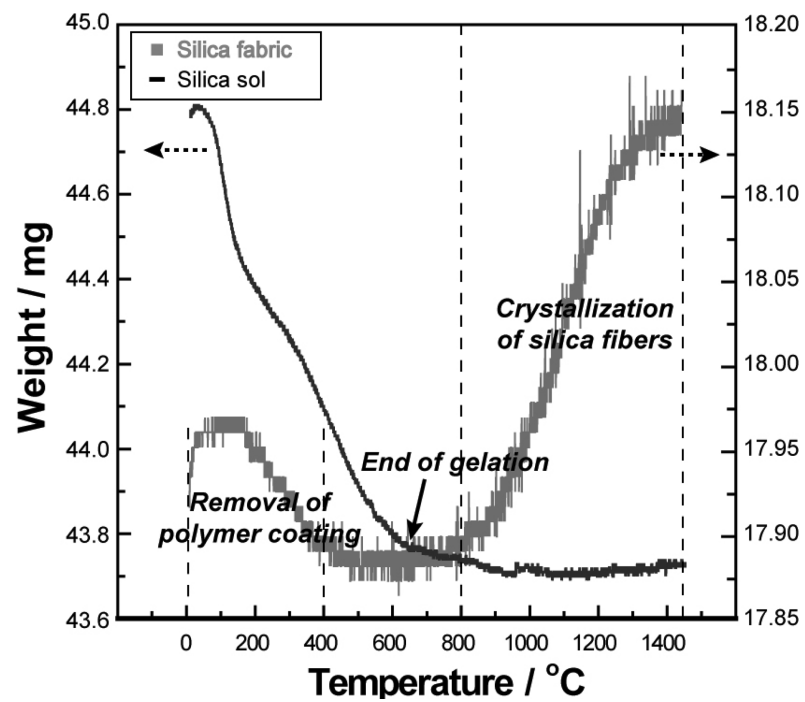

Fig. 1. Thermal gravitational analysis was conducted on both the silica fabric and the silica sol. The weight variation with increasing temperature was shown, where the gray rectangle indicates the silica fabric and the dark line does silica sol.

with increasing temperature in order to determine the heat treatment temperature. Thus, the TGA was conducted on both the silica fabric and the silica sol. The weight variation with increasing temperature was shown in Fig. 1 , where the gray rectangle indicates the silica fabric and the dark line does the silica sol. As shown in the figure, the weight of the fabric decreased upto $400{ }^{\circ} \mathrm{C}$ due to the removal of polymer coating on the fabric, and the weight increased sharply after $800^{\circ} \mathrm{C}$, which was caused by the crystallization of amorphous silica. In case of the silica sol, the weight decreased gradually upto approximately $650{ }^{\circ} \mathrm{C}$, where the gelation of sol completed. Thus, the heat treatment temperature of silica CFCCs was determined as $650{ }^{\circ} \mathrm{C}$.

\subsection{Characterizations of CFCCs}

The generation of porosities in the silica-silica composite from the sol-gel infiltration method is inevitable because the shrinkage occurs during the gelation of sol. In order to achieve dense composites, the procedure of sol-gel infiltration should be repeated. In this part, the relation between the repetition of infiltration and the relative density and mechanical properties of the composites will be discussed.

As shown in Fig. 2, The density of silica composites was different according as the number of repetition was set as 3,6 , and 9 times. The average density was $1.45 \mathrm{~g} / \mathrm{cm}^{3}$ in case of 3-times infiltration (3-SS), while it was 1.63 


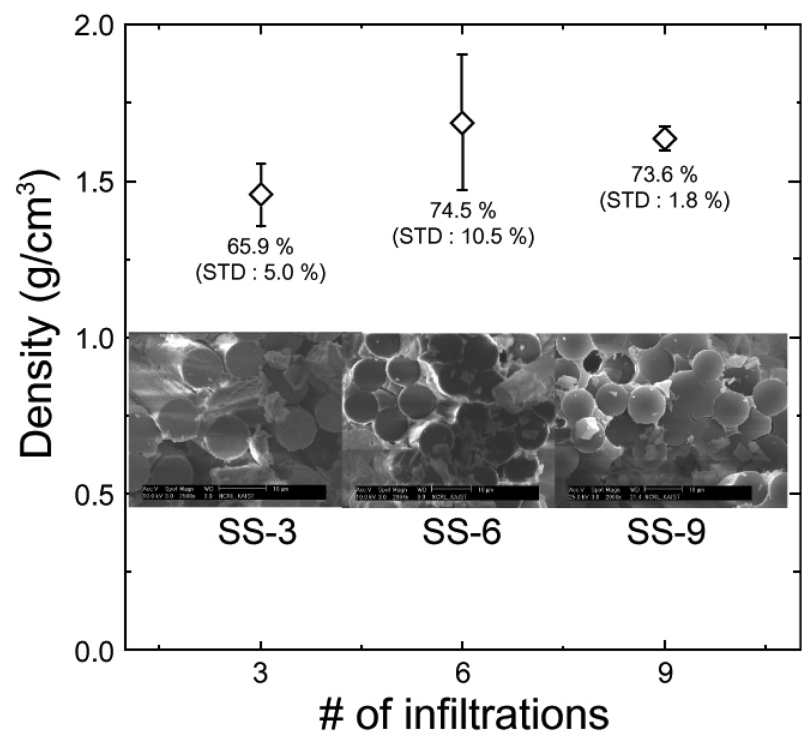

Fig. 2. The density of silica composites was different according as the number of repetition was set as 3,6, and 9 times. The more reliable composite can be achieved with increasing the repetition of infiltrations. As shown in the insets, the SEM micrographs of the fracture surfaces of silica CFCCs were characterized.

and $1.62 \mathrm{~g} / \mathrm{cm}^{3}$ in case of 6-times (6-SS) and 9-times infiltration (9-SS), respectively. The difference of the density between 6-SS and 9-SS specimens was not significant, however the standard deviation of the density in 9-SS was much lower than 6-SS. It means that the more reliable composite can be achieved with increasing the repetition of infiltrations. As shown in the insets of Fig. 2, the fracture surfaces of composites were characterized via SEM analysis. A few of gaps among individual fibers were observed in 3-SS specimens, while the gel filled up around individual fibers in 6-SS and 9-SS specimens. The silica-silica composite had microcracks in the silica matrix which is considered to cause the degradation of the strength.

The 4-point flextural test was conducted to investigate the effect of repetition of infiltrations on the mechanical properties such as flexural strength and elastic modulus. The stress-strain curves of silica CFCCs were shown in Fig. 3. In case of 3-SS (Fig. 3(a)), the curve showed that only matrix microcracking occurred during the flexural test due to the relatively low density to other specimens, whereas the curves of the 6-SS and 9-SS specimens showed representative stress-strain curve of CFCCs (Fig. $3(b)$ and (c)), that the curve was linear elastic up to $\sigma_{0}$ when the first matrix crack appears. From $\sigma_{0}$ onward, the
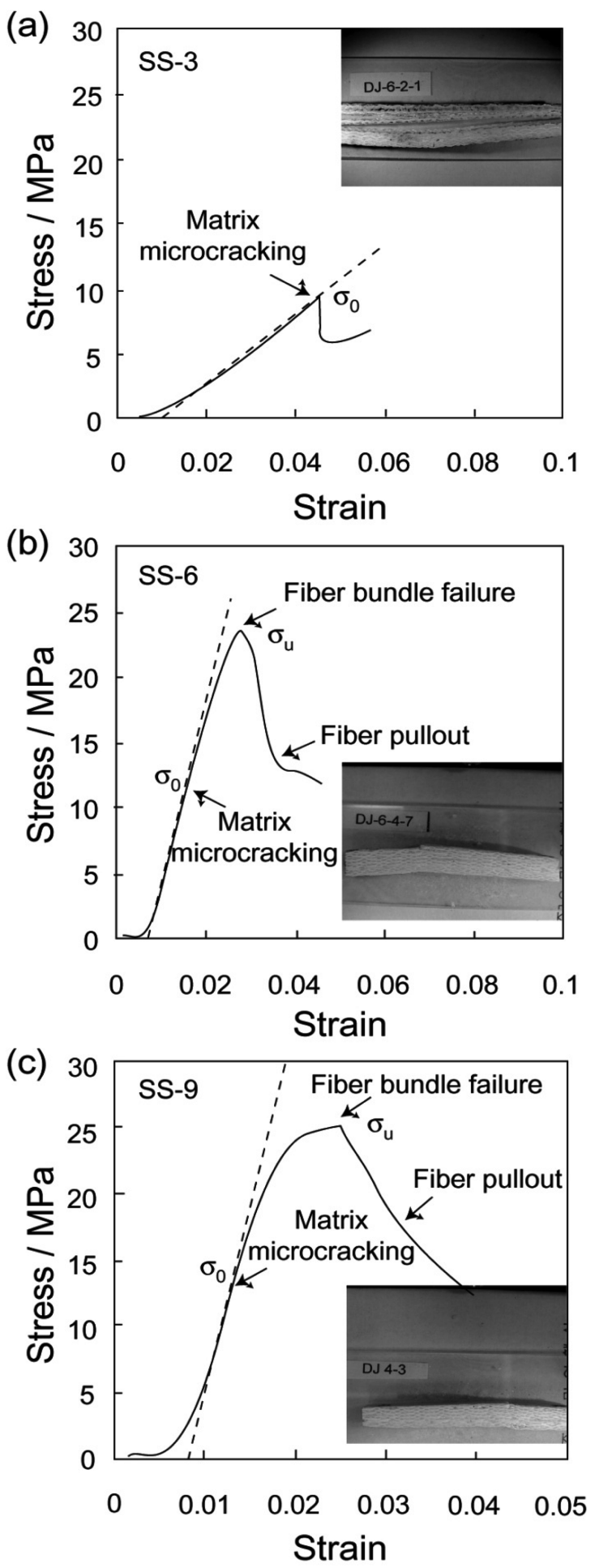

Fig. 3. The stress-strain curves of silica CFCCs were shown. (a) the curve showed that only matrix microcracking occurred during the flexural test due to the relatively low density to other specimens, (b) and (c) whereas the curves of the 6-SS and 9-SS specimens showed representative stress-strain curve of CFCCs, that the curve was linear elastic up to $\sigma_{0}$ when the first matrix crack appears. From $\sigma_{0}$ onward, the curve beame nonlinear until the ultimate stress of the fiber, $\sigma_{u}$ was reached. Straining past this point results in fiber pullout. 
curve became nonlinear until the ultimate stress of the fiber, $\sigma_{\mathrm{u}}$ was reached. Straining past this point resulted in fiber pullout. The appearance of silica composites after the test was shown in the insets of Fig. 3. The delamination among the fabrics occurred in case of 3-SS specimens (Fig. 3(a)), while it did not occurred in case of 6-SS and 9-SS specimens (Fig. 3(b) and (c)). It seems that when the density of composites is low, fabrics cannot be bonded each other, and the minimum repetition of infiltrations is necessary to avoid the delamination.

The elastic modulus and flexural strength of silica composites was shown in Fig. 4. As shown in Fig. 4(a), the average values of the elastic modulus were from 0.24 GPa for 3-SS specimens to 1.84 GPa for 9-SS specimens, and the low elastic modulus of 3-SS was due to the delamination under the loading. The more infiltrations repeated, the higher modulus of composites was achieved. The flexural strength of respective specimens was also shown in Fig. 4(a). The flexural strength of 3-SS specimens was from $3 \mathrm{MPa}$ to $15 \mathrm{MPa}$ due to the delamination, while those of 6-SS and 9-SS specimens were $21.5 \mathrm{MPa}$ and 23.2 MPa. The standard deviation of 9-SS was much lower than that of 6-SS, therefore it could be concluded that the reliable composites were achieved with increasing repetition of infiltrations. To investigate the relationship between the density of silica CFCCs and the mechanical properties, the density-flexural strength curves were plotted (Fig. 4(b)). As shown in the graph, there seemed to exist the minimum density value at which the delamination between the fabrics would not occur. In case that the density of silica CFCCs exceeded $1.55 \mathrm{~g} / \mathrm{cm} 3$, the flexural strength also exceeded $\sim 18 \mathrm{MPa}$.

\subsection{The minimum solid area (MSA) analysis}

Most of pore structures can be theoretically obtained from modifying degrees of bonding of various particles and packing with surrounding smaller pores, or using bubbles to form solid webs containing enclosd pores, or combinations of two cases. Thus, reasonable modelings of pore structures are regular stackings of several types of particles or bubbles. These models compose of stacking of spheres contained within regular polyhedral, often cubical, cells. Many of the models for the porosity dependence of physical properties of materials have been derived from using the above model structures to calculate actual solid cross-sectional areas. The fraction of the bulk property values as a function of the volume fraction porosity, $\mathrm{P}$, is
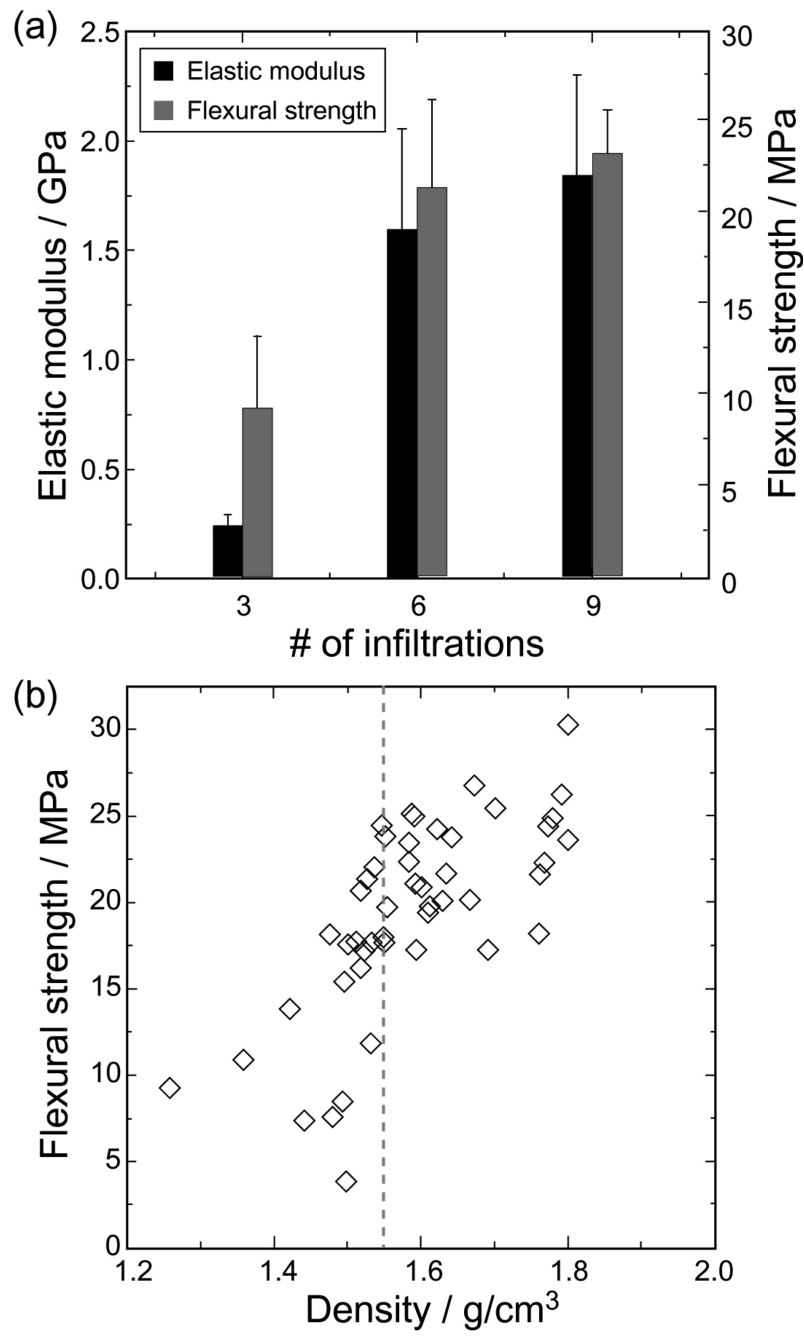

Fig. 4. The elastic modulus and flexural strength of silica CFCCs was plotted as a function of the repetitions of infiltrations, (a) the average values of the elastic modulus were from $0.24 \mathrm{GPa}$ for 3 -SS specimens to $1.84 \mathrm{GPa}$ for $9-\mathrm{SS}$ specimens, and the flexural strength of 3-SS specimens was from $3 \mathrm{MPa}$ to $15 \mathrm{MPa}$ due to the delamination, while those of 6-SS and 9-SS specimens were $21.5 \mathrm{MPa}$ and $23.2 \mathrm{MPa}$. (b) The flexural strength of silica CFCCs were plotted as a function of the density; there seemed to exist the minimum density value at which the delamination between the fabrics would not occur.

equated to either the minimum (i.e. average) fraction of solid area ${ }^{10-13)}$ of the total body area normal to a applying stress. Models of the minimum solid area analysis have been developed and used over the years for various individual pore structures, usually for mechanical properties such as elastic modulus and strength. The models were developed for uniform spherical pores, cubical pores, solid spherical particles (of simple cubic, orthorhombic, and rhombic stacking), and aligned cylindrical pores (stressed 
(a)

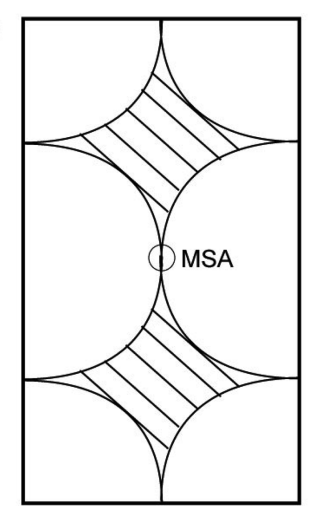

(b)

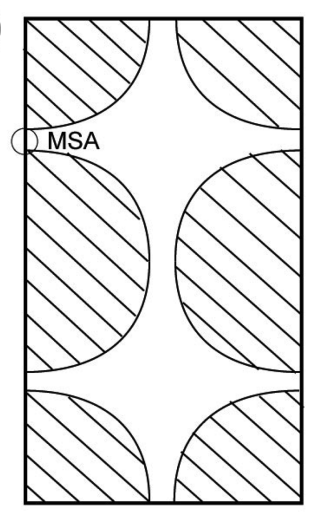

(c)

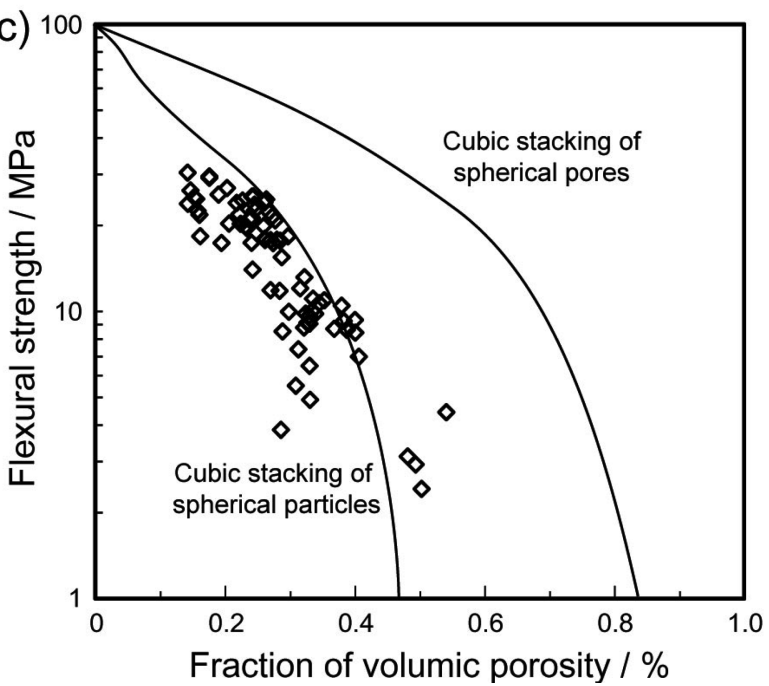

Fig. 5. The experimental density-flexural strength result was plotted with the curves with MSA models. (a) the schematics of the stacking of spherical particles (b) the schematics of the stacking of spherical pores (c) the experimental results showed similar tendency to the curve of the spherical particle model, which indicates that the minimum solid area in the silica CFCCs is highly low.

parallel or perpendicular to the pore axis). The minimum solid area and the mechanical properties of the stacking of spherical particle model have the simple exponential relation $\left(e^{-b P}\right)$ with a given $\mathrm{b}$ value. Subsequently, all of the other above-noted minimum solid area models for various pore structures were also similarly approximated by the exponential relation with various $b$ values, which correspond to given pore structures.

In this study, to investigate the dependence of pore structure on the flexural strength, the elastic modulus should be transformed to the flexural strength by the simple equation,

$\sigma=\varepsilon \times \mathrm{E}$

where $\varepsilon=$ strain, $\sigma=$ stress. The strain under flexural

testing condition could be calculated from the relationship between the strain and the displacement of crosshead of the INSTRON machine used,

$$
\varepsilon=\frac{6 h x}{L^{2}}
$$

where $\mathrm{h}=$ specimen thickness, $\mathrm{x}=$ displacement of crosshead, and $\mathrm{L}=$ outer span. The experimental density-flexural strength result was plotted with the curves, Which were MSA models of the stacking of spherical particles (Fig. 5(a)) and spherical pores (Fig. 5(b)) where the strength of the fully dense silica monolith was assumed as $100 \mathrm{MPa}$ (Fig. 5(c)). As shown in the graph, the experimental results showed similar tendency to the curve of the spherical particle model, which indicates that the minimum solid area in the silica CFCCs is highly low. Because the material composed with the stacking of spherical pores has higher elastic modulus as well as flexural strength than the the material with the stacking of spherical particles, so that if the matrix structure can be tailored from the separated structure to the network structure, the silica CFCCs with improved mechanical properties would be achieved.

\section{Conclusions}

2-dimensional silica-silica continuous fiber-reinforced ceramic-matrix composites (CFCCs) were successfully fabricated by sol-gel infiltration method. From the flexural strength test, the silica composites showed the representative stress-strain curves of typical CFCCs and the minimum density value, at which the delamination between the fabrics would not occur, could be found with the evidences of strength degradation via SEM analysis. Applying the minimum solid area (MSA) analysis of the porous structure, the experimental results showed the similar tendency to the porosity-strength curve of the stacking of spherical particles model, which indicates that the minimum solid area in the silica CFCCs is highly low. Thus, it can be concluded that if the matrix structure can be tailored from the separated structure to the network structure, the silica CFCCs with improved mechanical properties would be achieved.

\section{Reference}

1. J. P. Brazol, R. Fenton, J. Roetling and R. Tanzilli, Final 
Report of Millimeter Wave Hardened Antenna Window Materials Development, p.13, Army Materials and Mechanics Research Center, Massachusetts, USA (1979).

2. M. Ruhle and A. G. Evans, Prog. Mater. Sci., 33, 85 (1989).

3. R. S. Russell-Floyd, B. Harris and R. G. Cooke, J. Am. Ceram. Soc., 76, 2635 (1993).

4. M. N. G. Nejhad, M. V. Chandramouli and A. Yousefpour, J. Comp. Mat., 35, 2207 (2001).

5. N. E. Prasad, S. Kumari, S. V. Kamat, M. Vijayakumar and G. Malakondaiah, Eng. Frac. Mech., 71, 2589 (2004).

6. N. E. Prasad, D. Loidl, M. Vijayakumar and K. Kromp.
Scripta Mater., 50, 1121 (2004).

7. R. W. Rice, Porosity of Ceramics, p. 49, ed., R.W. Rice, Marcel Dekker, Inc., New York, USA. (1997).

8. R. E. Fisher, C. Burkland and W. E. Bustamante, Cer. Eng. \& Sci. Proc., 6, 806 (1985).

9. J. Jamet, J. R. Spann, R. W. Rice, D. Lewis II and W. S. Coblenz, Cer. Eng. \& Sci. Proc., 5, 443 (1984).

10. F. P. Knudsen, J. Am. Ceram. Soc., 42, 376 (1959).

11. F. P. Knudsen, J. Am. Ceram. Soc., 45, 94 (1962).

12. R. W. Rice, J. Am. Ceram. Soc., 59, 536 (1976).

13. R. W. Rice, J. Mater. Sci., 31, 102 (1996). 